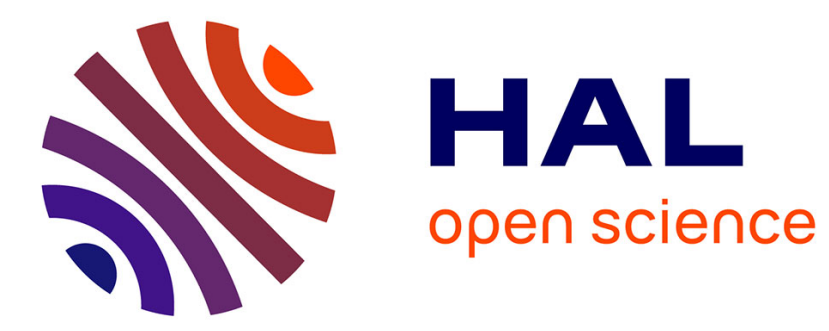

\title{
Terrassiers, kiosquiers et bouquinistes à Rabat et à Casablanca
}

\author{
Anouk Cohen
}

\section{To cite this version:}

Anouk Cohen. Terrassiers, kiosquiers et bouquinistes à Rabat et à Casablanca. Ethnologie française, 2017, Gens de commerce, 165 (165), pp.23-36. 10.3917/ethn.171.0023 . hal-01632130

\section{HAL Id: hal-01632130 \\ https://hal.parisnanterre.fr/hal-01632130}

Submitted on 15 Dec 2020

HAL is a multi-disciplinary open access archive for the deposit and dissemination of scientific research documents, whether they are published or not. The documents may come from teaching and research institutions in France or abroad, or from public or private research centers.
L'archive ouverte pluridisciplinaire HAL, est destinée au dépôt et à la diffusion de documents scientifiques de niveau recherche, publiés ou non, émanant des établissements d'enseignement et de recherche français ou étrangers, des laboratoires publics ou privés. 


\title{
Terrassiers, kiosquiers et bouquinistes à Rabat et à Casablanca Circulation du savoir et matérialité du livre
}

Anouk Cohen

Laboratoire d'ethnologie et de sociologie comparative

\begin{abstract}
RÉSUMÉ l'écrit dans ces villes marocaines.

Anouk Cohen

LESC (CNRS, UMR 7186)

Maison Archéologie \& Ethnologie, René-Ginouvès

21, allée de l'Université

92023 Nanterre cedex

anouk.cohen@mae.u-paris10.fr
\end{abstract}

À Rabat et à Casablanca, capitales administratives et économiques du Maroc, une grande partie de la production éditoriale, des journaux et des magazines est écoulée directement dans la rue, sur les trottoirs et dans les kiosques. Le nombre élevé de kiosquiers, terrassiers et bouquinistes (suivant la terminologie employée localement) indique que leurs commerces ne constituent pas un mode de diffusion marginal contrairement à ce que leur aspect précaire pourrait laisser croire. Le but de cet article est de mieux cerner le rôle crucial que ces marchands de rue, souvent analphabètes ou illettrés, jouent dans la circulation de

Mots-clefs : Marchands de livres. Circulation du savoir. Matérialité. Analphabétisme. Maroc.

À Rabat et à Casablanca, capitales administrative et économique du pays, les grandes artères séparant la médina (en arabe, la partie ancienne d'une ville par opposition au quartier moderne) de la ville nouvelle offrent une scène particulière pour la vente des livres : installés sur des feuilles de carton posées sur les trottoirs, des ouvrages de tous types (Coran, livres religieux, romans, récits, essais, ouvrages techniques, etc.) sont ouverts, consultés et achetés. Tandis qu'il n'existe qu'une poignée de librairies, c'est parfois tous les vingt mètres que l'on trouve ces "librairies de trottoirs" [Gonzalez-Quijano, 2002 [1998] ; El-Alaoui, 2006]. Au coin des rues les plus fréquentées du centre-ville, chacun de ces commerces dessine une mosaïque de couleurs et contribue à singulariser l'aspect de ces avenues en les revêtant de teintes particulières. Les journaux et les livres exposés dans la rue participent, par leur présence massive, de la transformation de l'espace public en espace marchand.

De ces premières observations deux constats peuvent être formulés : d'une part, l'imprimé investit largement la rue et l'espace public ; d'autre part, la commercialisation du livre s'effectue par le biais d'une multitude de marchands qui officient dans divers espaces de vente: les libraires, spécialisés dans la vente de livres, disposent de magasins dotés d'une vitrine et d'une porte ; les buralistes ${ }^{1}$ vendent des produits variés tels que des journaux, magazines, cigarettes, livres, etc. depuis une petite boutique également dotée d'une porte; les kiosquiers vendent la presse et les livres depuis un édicule ouvert, débordant sur la rue ; les "terrassiers" (suivant la terminologie employée par mes interlocuteurs) vendent des livres, des journaux et des magazines depuis des étals installés à même le trottoir. Les bouquinistes, installés principalement dans les souks et les médinas, possèdent de petites boutiques en forme de $U$ sur une surface de 10 mètres carrés, refermées ou non par un comptoir en bois. Ils y commercialisent des livres et des revues d'occasion. Ainsi, on différencie deux types de commerces : d'une part ceux ouverts sur la rue et les marchés (kiosques, terrasses et boutiques de bouquinistes), d'autre part, les commerces qui constituent des espaces de vente fermés (buralistes et librairies). C'est aux premiers que cette analyse est consacrée.

Contrairement à ce que leur aspect précaire pourrait laisser croire, le nombre élevé de terrassiers, kiosquiers et bouquinistes montre que leurs commerces ne constituent pas un mode de diffusion marginal. Situés dans 
des endroits très fréquentés, au coin des avenues centrales comme les boulevards Mohammed V et Hassan II où se situent la gare, le bureau de poste et de nombreux commerces, ces espaces faciles d'accès sont des lieux d'attraction autour desquels, aux heures de pointe, une foule gravite toujours. Le but de cet article est de comprendre le rôle crucial que ces marchands de rue jouent dans la circulation de l'écrit à Rabat et à Casablanca.

À cette fin, j'ai étudié qui ils sont, comment ils travaillent et quels types de rapports ils entretiennent avec l'imprimé, en particulier le livre. Commencer par décrire les trajectoires de terrassiers, kiosquiers et bouquinistes m'a conduite à examiner leurs pratiques de travail et leurs compétences professionnelles ainsi que le fonctionnement de leurs espaces de vente - la question de l'emplacement, de la structure physique, de l'installation des livres, des horaires et des prix, comme le genre d'atmosphère qui y règne. Il s'agit dès lors de s'intéresser aussi bien aux marchands de rue qu'aux acteurs avec lesquels ils entrent en relation, non seulement les libraires mais aussi les éditeurs et les clients. Enfin, une attention particulière a été portée à la manière dont les kiosquiers, terrassiers et bouquinistes, souvent analphabètes ou illettrés, donnent accès à l'écrit aux personnes qui n'en sont pas familières et offrent la possibilité à des auteurs censurés de faire connaitre leur pensée avec les moyens dont ils disposent ; c'est-à-dire, en exploitant les matérialités physiques du livre pour structurer leur commerce et procéder à la vente d'ouvrages.

\section{Retracer le parcours du livre. Une ethnographie}

Cet article est le fruit d'une enquête ethnologique effectuée à Rabat et à Casablanca au cours de l'année 2006-2007 et de plusieurs séjours réalisés en 2008, 2009, 2010 et 2015. Durant neuf ans, j'ai rencontré et interrogé un grand nombre de terrassiers, kiosquiers et bouquinistes. Parallèlement à ces entretiens, ma présence sur le terrain m'a permis d'observer les pratiques du livre dans la rue et les points de vente pendant près d'un an et demi. J'ai choisi de suivre le livre dans ses relations avec ses différents publics afin de saisir au mieux cet imprimé dans tous ses états 2 . Retracer le parcours du livre depuis la rue m'a amenée à porter une attention centrale aux marchands qui officient dans cet espace, aux manières dont la rue structure, voire définit, leurs pratiques de travail et enfin aux gestes qu'ils exécutent pour distribuer leurs livres et contribuer, à leur niveau, à la circulation de l'écrit dans la ville. Cette réflexion s'inscrit dans le prolongement de la démarche entreprise dans le second volume des Lieux de savoir dirigé par Christian Jacob, qui vise à "réduire l'hiatus entre les pratiques de la main et les pratiques de l'intellect, ou plus exactement à penser les liens qui les relie » [Jacob, 2011: 20].

\section{- Trajectoires}

\section{Marchands, clients et espaces}

À Rabat et à Casablanca, comme ailleurs au Maroc et dans le monde arabe, une grande partie de la production éditoriale, des journaux et des magazines est écoulée directement dans la rue, sur les trottoirs et dans les kiosques. À proximité de ces commerces, se rencontrent des installations plus modestes, seulement composées de quelques feuilles de carton posées à même le sol : les terrassiers (photo 1). Alors que les kiosquiers et les terrassiers s'adressent principalement à un lectorat peu fortuné et peu familier de l'écrit, lisant davantage la presse que les livres, les libraires et les buralistes, quant à eux, destinent leurs ouvrages à des lecteurs plus réguliers, voire assidus, prêts à dépenser jusqu'à $120 \mathrm{dh}$ (prix des livres importés du Moyen-Orient) et 250 dh (ouvrages en provenance de France) pour acquérir un livre sur un thème précis, souvent en lien avec leur profession. Ces lecteurs, qui sont généralement enseignants, journalistes, avocats, médecins, etc. se rendent principalement dans deux espaces de vente distincts suivant la langue dans laquelle ils préferent lire : les librairies spécialisées dans le livre d'expression arabe ou les librairies spécialisées dans le livre d'expression française $e^{3}$. Alors que les premières proposent majoritairement des livres en provenance du Liban et d'Egypte, les secondes, réservées à une élite maitrisant bien le français, fondent leur prestige sur les livres importés de France dont les prix élevés sont inabordables pour la grande majorité des Casaouis et des Rabatis (habitants de Casablanca et de Rabat) ${ }^{4}$.

Cette bipolarité se manifeste également dans la répartition spatiale des maisons d'édition et des librairies. Suivant leur spécialité linguistique, elles se situent dans des quartiers spécifiques: tandis que les sociétés du livre arabophone se concentrent dans le quartier des Habous où tous types de produits - vêtements, ustensiles de cuisine, fruits et légumes, livres, etc. - sont vendus en gros, celles du livre francophone prennent majoritairement place dans le centre-ville et dans le quartier du Maârif qui abritent un nombre considérable de commerces inscrits dans la culture de l'ancienne métropole : cafés (Café de Paris), restaurants (le Petit Poucet, autrefois fréquenté par Jean Genet), et librairies (le Livre-Service, la Librairie de France, la Librairie Farraire, la Librairie des Sciences, etc.).

C'est à proximité de ces librairies que les kiosquiers et les terrassiers se sont implantés au fil du temps. 


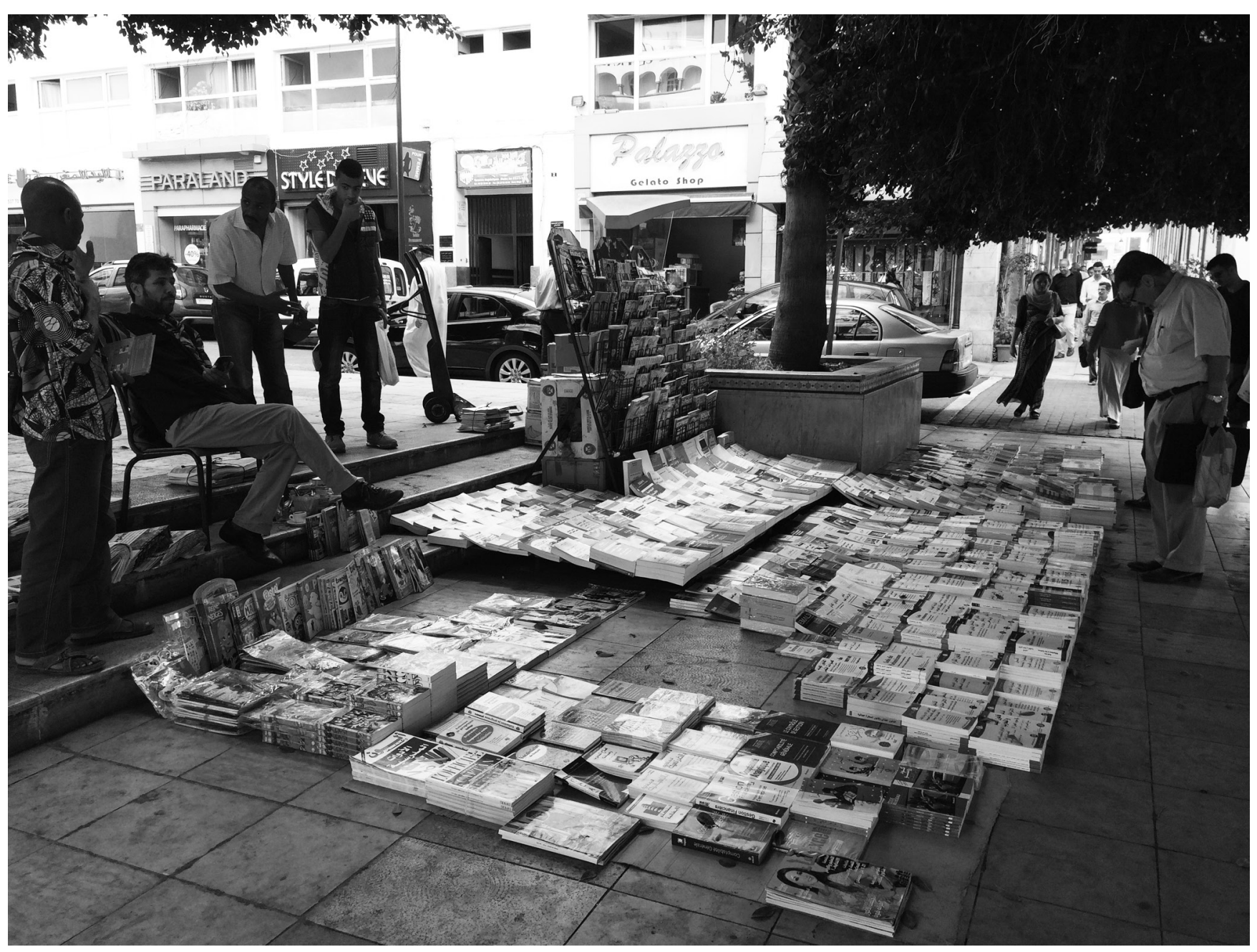

Photo 1 - Terrassier de l'avenue Hassan II à Rabat (photo de l'auteur, 5 octobre 2015).

La présence d'une population étrangère, principalement française et espagnole, dans cette zone a été déterminante dans ce processus. Elle composait, en effet, la clientèle privilégiée des premiers terrassiers, kiosquiers et bouquinistes. Ces vendeurs à la sauvette leur proposaient livres, journaux, magazines, bandes dessinées. Autant d'imprimés qui ont fini par conquérir cet espace toujours marqué par la présence du livre. Plus que de constituer des marqueurs de l'histoire coloniale et urbaine, la présence de ces marchands souligne la relation étroite qui existe entre espace et objet. Ils " font lieu " - suivant l'expression de Christian Jacob [2007 : 17] - à travers leurs boutiques, kiosques et terrasses.

\section{"J'ai toujours fait ça!»}

La plupart de ces marchands de rue sont devenus commerçants de livres parce que leur père ou leur oncle leur a transmis sa boutique et son savoir-faire. Ils ont appris le métier sur le tas, par imitation et répétition des gestes de leurs aînés. Les métiers de commerce du livre se transmettent de génération en génération, principalement entre hommes. Peu de femmes vendent des livres dans les kiosques et sur les trottoirs alors qu'elles sont nombreuses à travailler dans les librairies. Tandis que les hommes se chargent de passer commande auprès des distributeurs, d'accueillir les éditeurs et les auteurs qui souhaitent déposer leurs ouvrages, elles accueillent 
les clients, les renseignent et encaissent leurs achats. Les vendeuses sont souvent la fille, la sœur ou la nièce du propriétaire. Pour elles aussi, le métier est affaire de famille. Cet héritage explique en partie pourquoi ces marchands n'ont généralement pas d'attachement particulier au livre. S'ils travaillent " dans le livre ", comme la plupart disent, c'est souvent parce qu'ils ont «toujours fait ça! " ou parce qu' «il n'y a pas de travail ».

Pour Ali, bouquiniste à Derb Ghellaf, vaste marché réputé pour ses produits informatiques (téléphones portables, ordinateurs, logiciels divers, DVD, téléviseurs, baladeurs numériques, etc.) et la grande variété de ses marchandises (vêtements, lingerie, literie, vaisselle, fruits et légumes, livres d'occasion, etc.), " c'est mieux de travailler dans le livre plutôt que de vendre des légumes, c'est pas un métier ça!"

Par ailleurs, vendre des livres au souk ne lui déplaît pas. Il aime Derb Ghellaf, les habitudes de travail et surtout la proximité qui relie les différents commerçants du marché. Il lui est par exemple facile de confier son stand à un confrère lorsqu'il doit s'absenter. Ali apprécie davantage l'environnement dans lequel son commerce prend place que la marchandise autour de laquelle il est organisé. Tandis qu'il affiche un certain désintérêt pour les livres, Larbi, qui tient la boutique d'à côté, se présente au contraire comme un lecteur régulier et établit un lien direct entre son travail et sa passion pour le livre.

Alors qu'une grande partie des bouquinistes, kiosquiers et terrassiers sont analphabètes ou illettrés, d'autres, comme Larbi ou Hassan, célèbre kiosquier de l'avenue des Far à Casablanca, sont de grands lecteurs régulièrement consultés par de fidèles clients pour connaitre les nouveautés. Hakim qui habite à proximité du kiosque de Hassan s'y rend chaque semaine pour discuter avec lui des «livres à lire ». "Avant de rencontrer Hassan, je ne lisais pas, poursuit-il. À la maison, personne ne lisait. Puis avec Hassan et d'autres clients on discute de l'actualité, des unes des journaux, on se retrouve là et on parle. Hassan nous fait connaître des livres parus en Egypte et au Liban. Du coup, ça m'a donné envie de lire ». Ainsi, la relation au métier de kiosquier, terrassier ou bouquiniste differe d'un individu à l'autre.

\section{- Devenir bouquiniste par opportunisme}

L'exercice de ce dernier métier peut également être circonstanciel. Larbi, que l'on nomme le hâjj ${ }^{5}$, âgé d'une soixantaine d'années, a ouvert sa boutique en 1958. Quand je lui demande ce qui l'a amené à exercer ce métier, il répond:

Tu sais, en 54, on m'a viré de l'école, parce que je ne voulais pas lire les livres de l'école, je ne voulais pas apprendre, je lisais des BD, je ne faisais que ça ! Le directeur m'a convoqué avec mes parents et m'a dit de partir. Alors j'ai commencé à vendre des BD au quartier du Maârif à des Français, des Espagnols, des Juifs, des Italiens, etc. D'abord, je vendais des livres dans la rue du Jura après $\mathrm{j}$ 'ai ouvert une boutique dans le Souk de Derb Ghallef. À leur départ beaucoup de Français m'ont vendu et parfois même donné leurs livres.

Les années 1970 furent marquées par une vague de départs des étrangers après l'entrée en vigueur du plan de marocanisation qui visait à définir un nouveau partage entre les capitaux étrangers et nationaux de façon à associer l'oligarchie aux grandes affaires industrielles et commerciales étrangères, plus particulièrement françaises. Dans le cadre de ce plan, les étrangers se voyaient dans l'obligation de trouver des partenaires marocains avec qui gérer leurs sociétés. Beaucoup ont refusé et préféré quitter le Maroc après avoir vendu ou donné leurs biens. Larbi ne se souvient pas des raisons qui ont poussé ces Français à lui vendre ou céder leurs livres à lui plutôt qu'à un autre. Sont devenus bouquinistes ceux vers qui les Français se sont tournés pour vendre leurs bibliothèques dans le but d'alléger leurs malles pour le départ en bateau. C'est pourquoi Larbi ne vend que des livres en français bien que, déclare-t-il, il ne lise "que l'arabe ». La musique en langue arabe diffusée par le poste de radio installé à l'entrée marque un décalage net avec les livres rangés sur les étagères de sa boutique : d'anciennes éditions françaises, en particulier J'ai lu, Livres de poche et Folio.

La prédominance de ces collections tient aussi à la politique d'acquisition de Larbi qui s'approvisionne auprès de soldeurs dont la marchandise est principalement constituée d'ouvrages français sauvés du pilon et envoyés au Maroc. Ces soldeurs organisent périodiquement des ventes sur les marchés à Casablanca où se rendent régulièrement les revendeurs des souks en gros de Derb Ghallef ou de Derb Omar. Les bouquinistes achètent les livres entre 5 et 10 dh pièce pour les revendre entre 15 et 30 dh suivant les collections : les Livres de poche et les Pocket coûtent $15 \mathrm{dh}$, les Folio et les J'ai Lu $20 \mathrm{dh}$, les J'ai Lu "rouge » (correspondant à la collection "Aventure et passion ") $30 \mathrm{dh}$; les livres récents sont vendus $30 \mathrm{dh}$ quelle que soit la collection 
dans laquelle ils sont édités. Ainsi, Larbi vend-il les livres qu'il trouve sur les marchés en s'adaptant à l'offre des soldeurs sans forcément avoir au préalable défini une politique d'acquisition. Pour lui, c'est surtout la question du stock qui est cruciale : comme sa boutique est petite, Larbi ne peut pas y entreposer beaucoup d'ouvrages. C'est donc en fonction de l'espace disponible dans son magasin et chez lui qu'il décide ou non de se rendre sur les marchés des soldeurs. Voilà donc comment un stock qui lui vient de façon plus ou moins aléatoire (un marchand aurait pu le prendre avant lui, il aurait pu ne pas avoir l'espace disponible pour le stocker ou de quoi l'acheter au moment où il a été mis sur le marché) oriente son commerce. L'accès au stock de livres soldés a permis à Larbi de découvrir l'univers des livres de littérature française.

Cependant, son activité ne s'articule pas autour de ce seul et unique stock. Un processus d'enchaînements

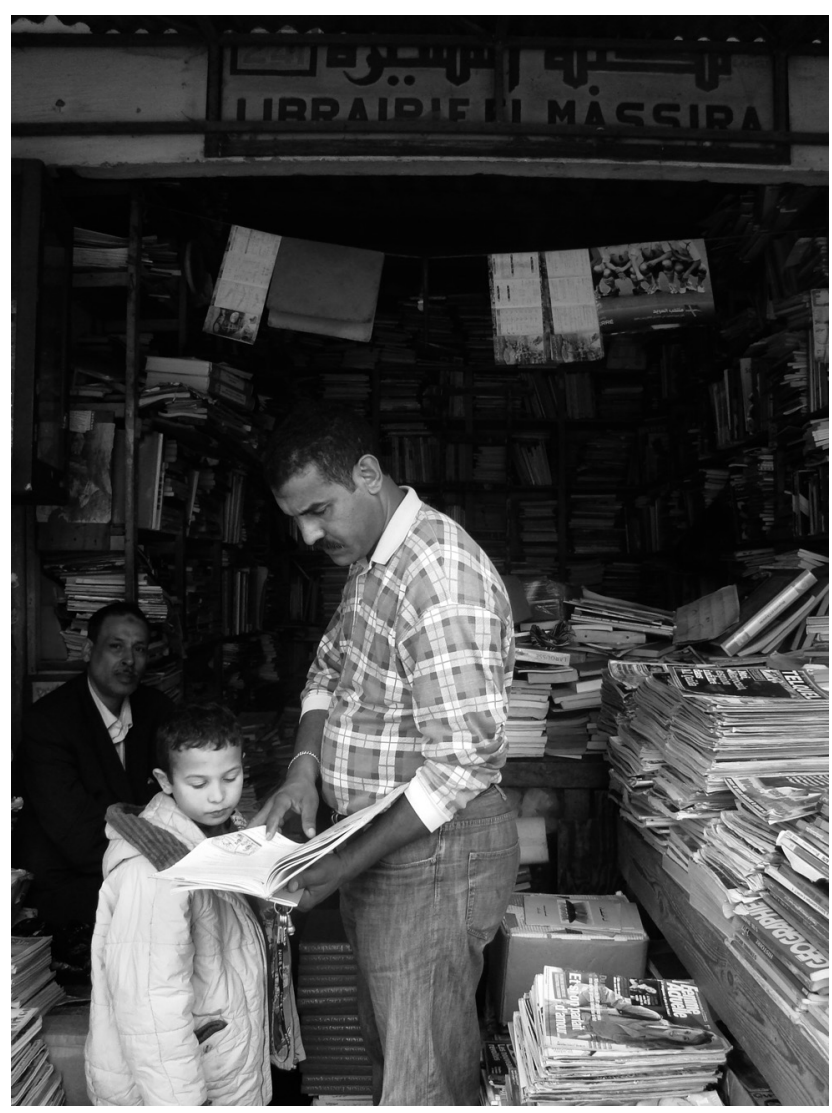

Photo 2 - Larbi, bouquiniste à Derb Ghellaf (photo de l'auteur, 14 avril 2007). complexes peut être à l'origine d'une situation marchande spécifique car les trajectoires professionnelles sont orientées soit par la nécessité de faire un choix en rapport avec les attentes de la clientèle, soit par d'autres occasions, plus hasardeuses. Les bouquinistes, en effet, sont en permanence à la recherche d'ouvrages et peuvent en acheter directement aux particuliers. Lors des discussions que j'ai pu avoir avec eux, beaucoup m'ont demandé si j'avais des livres à leur vendre. Larbi, par exemple, recherche de préférence des ouvrages d'histoire sur le Maroc des XVIII ${ }^{\mathrm{e}}$ et $\mathrm{XIX}^{\mathrm{e}}$ siècles : "Des livres qui coûtent cher. Comme les encyclopédies." Il sait que ce type d'ouvrages est très apprécié par une catégorie de clients, en l'occurrence des collectionneurs, soucieux d'enrichir leur bibliothèque avec de beaux livres et des encyclopédies. Cette clientèle, néanmoins, est loin d'être la plus importante et, autour de leurs stands, j'ai surtout vu les nombreux libraires qui se rendent chaque semaine - généralement le dimanche lorsque leur boutique est fermée - à Derb Ghallef pour acheter certaines collections épuisées, mais toujours très demandées par leurs clients, comme les "J'ai lu rouge ". À cette fin, il leur arrive également de s'approvisionner auprès des kiosquiers et des terrassiers ou de leur vendre les livres qu'ils ne parviennent pas à écouler à des prix bon marché. J'y ai également vu des personnes qui cherchent à acquérir des livres de littérature française (généralement ceux inscrits au programme scolaire) mais dont le niveau de vie ne permet pas de les acheter neufs en librairie. En outre, beaucoup d'entre eux déclarent préférer se rendre chez les bouquinistes de Derb Ghallef car ils pratiquent le prêt.

\section{Censure et tours de mains}

C'est le plus souvent chez les kiosquiers et davantage encore chez les terrassiers qu'on parvient à dénicher les rares livres rédigés en arabe dialectal marocain (dârija) et en berbère (amazigh). Jusqu'à une période récente, les libraires n'en proposaient jamais en raison de l'interdit qui a longtemps pesé sur ces écrits. Avant l'officialisation de l'amazigh comme seconde langue du pays en juin 2011, à la suite des soulèvements populaires qui avaient commencé en février, le pouvoir, au Maroc comme ailleurs, avait essayé par tous les moyens de promouvoir la notion d'unicité dans le domaine linguistique ${ }^{6}$. En raison du monolithisme des pays arabes, en particulier ceux du Maghreb, destiné 
à promouvoir les processus de création nationale et à les ancrer au sein d'une culture et d'une identité pan arabes, la pluralité linguistique a longtemps été stigmatisée. Elle apparaissait comme éminemment transgressive, accusée de véhiculer des valeurs antinationales. C'est pourquoi l'État a cherché à restreindre l'emploi de ces langues en interdisant leur introduction dans la sphère publique [Pouessel, 2008]. Cet interdit persiste jusqu'à aujourd'hui concernant la dârija [Cohen, 2011]. D'après les auteurs qui distribuent eux-mêmes leurs ouvrages rédigés en arabe dialectal marocain, les libraires les refusent presque toujours, contrairement à certains buralistes auprès de qui il est possible d'en placer. C'est aussi le cas des livres qui traitent de sujets sensibles tels que la personnalité du roi, la sexualité et le Sahara occidental ${ }^{7}$ ou des ouvrages qui proposent un point de vue critique ou fondamentaliste sur la religion ${ }^{8}$.

En effet, les libraires sont soumis à une réglementation stricte, les obligeant à déclarer toute nouvelle acquisition d'ouvrages au ministère de la Communication qui les informe des livres pouvant être interdits à la vente. Au contraire, les étalages de la rue et des marchés échappent davantage au contrôle et peuvent offrir à l'occasion des ouvrages qui ne mentionnent ni éditeur, ni dépôt légal. Ainsi Jamal, libraire au Livre service à Casablanca, constate :

À chaque fois que j'importe du livre, il me faut un visa du ministère de la Communication qui peut censurer s'il le veut. Avant la censure était plus importante qu'elle ne l'est aujourd'hui. Parfois, je recevais même des procès-verbaux envoyés directement par le commissaire divisionnaire qui interdisait la vente de certains livres. Je devais alors signer ces procès-verbaux, les renvoyer et en garder une copie. À l'époque, on en recevait au moins trente par an. Le dernier en date que j'ai reçu est de 2002, et depuis rien. Tout ce qui touche à la personnalité du roi ou au Sahara c'est interdit. Par exemple, on ne devait accepter aucun dictionnaire qui ne faisait pas figurer le Sahara sur la carte du Maroc. Ou alors, certains livres sur la religion. Et maintenant, c'est plus libre, mais vous ne trouverez pas un Versets sataniques de Salman Rushdie au Maroc ni de la pornographie ! Ça surtout pas! Mais maintenant je sais quoi importer, je sais ce qui est interdit. C'est de l'autocensure.

Chez les kiosquiers et les terrassiers, plutôt que les livres, ce sont davantage les journaux et les magazines qui sont contrôlés. Les libraires s'adressent à ces points de vente pour écouler des ouvrages qu'ils auraient acquis mais que l'autorité désapprouve a posteriori. C'est ensuite aux kiosquiers et terrassiers de les vendre avec plus ou moins de précautions. Généralement, ils ne les exposent pas sur leurs étalages et attendent que des clients les leur demandent pour les extraire de leur sacoche ou, pour les kiosquiers, d'un coin dissimulé de leur édicule. Libraires, terrassiers, kiosquiers et bouquinistes sont donc compétitifs tout en restant solidaires. Ils travaillent en étroite collaboration pour diffuser l'écrit. L'inscription des kiosquiers et des terrassiers dans la rue, où l'intensité des déplacements et des échanges gêne la surveillance, facilite ce type de pratiques et rend possible le commerce de tous types de produits, réglementés ou non. Néanmoins, le fonctionnement des kiosques repose aussi sur un certain nombre de normes. L'exercice de cette activité nécessite notamment la possession d'un " agrément " payant - Mohamed, par exemple, l'a hérité de son père. Ce titre de propriété soumet son détenteur à l'État qui peut à tout moment, en revenant sur ce titre, saisir la marchandise. De plus, il lui faut payer une «patente d'environ 1500 dh par an ».

Les terrassiers, quant à eux, travaillent de manière tout à fait informelle : à la différence de Mohamed, Hassan, terrassier, ne paie aucune patente. "C'est plutôt moi qui rends service à vendre les journaux des partis politiques et du Royaume !", déclare-t-il. Ainsi, les terrassiers n'ont-ils pas de comptes à rendre à l'État et bénéficient-ils d'une marge de liberté plus grande que les autres marchands. C'est souvent par eux que transite la littérature censurée. Et à Rabat et à Casablanca, comme ailleurs, la rue est l'espace où circulent et peuvent s'acquérir les textes interdits. Comme les colporteurs en France au XIX ${ }^{\mathrm{e}}$ siècle, qui faisaient le lien entre les peuples et les libraires qui parvenaient grâce à eux à mieux connaître le goût du public [Chartier et Lusebrink, 1996], les terrassiers et les kiosquiers rendent possible la diffusion des textes censurés à l'ensemble de la société.

\section{La rue comme espace de vente}

Ainsi que nous l'avons dit plus haut, les bouquinistes de Derb Ghallef comme ceux d'autres souks proches d'universités, offrent un système de prêt. Il s'agit d'une pratique initiée par les premiers marchands de rue, à laquelle adhèrent aussi certains kiosquiers. Le principe est simple : une fois que le client a acheté un livre 
au prix normal, il pourra, s'il le souhaite, l'échanger contre un autre pour 2 dh dans le cas d'un livre de poche, ou 5 dh s'il s'agit d'un "J'ai lu rouge ", collection la plus recherchée. Il n'est pas tenu de le rendre avant une certaine date: "Quand tu as fini de le lire, tu reviens ", dit souvent Larbi qui ne prend pas en note les ouvrages sortis et les dates auxquelles ils ont été empruntés : "Ça tourne tout seul ", déclare-t-il. Cette pratique attire principalement des jeunes au faible revenu qui préferent les emprunter plutôt que de les acheter. Le livre "se passe " donc de main en main, circule jusqu'à revenir au vendeur; il fait donc l'objet de transactions inscrites dans des circuits autres que ceux de l'économie classique.
Ainsi, Mohamed est un kiosquier réputé pour le prêt qu'il pratique au même tarif que les bouquinistes.

Son kiosque est ouvert tous les jours sauf le dimanche, de 7 heures à 21 heures. Tandis qu'il travaille les matinées, son neveu est en charge de la boutique les après-midi. Mohamed précise néanmoins que "le travail commence dès quatre heures du matin", lorsque les camions de Sochepress, principale société de distribution marocaine, livrent journaux, magazines et ouvrages. Au fil de la journée, ce sont les auteurs et les éditeurs qui viennent leur déposer des livres. Cependant, Mohamed veille à ne pas en acquérir un trop grand nombre car "ils se vendent moins bien que les magazines et les journaux ». En outre, il préfere

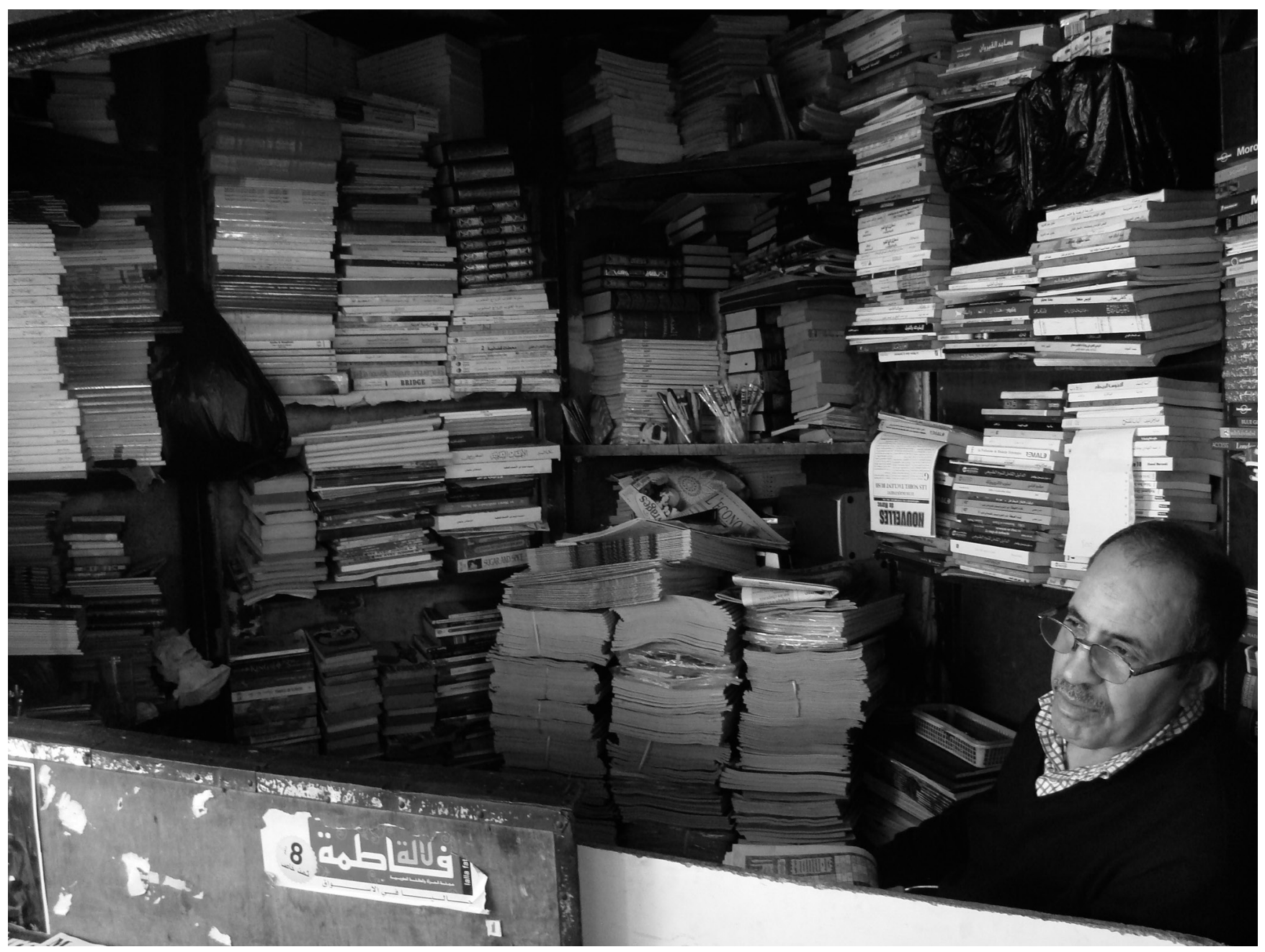

Photo 3 - Mohamed, kiosquier dans l'avenue Mohammed V à Casablanca (photo de l'auteur, 15 mars 2007). 
saisir les tendances suivant la demande de ses clients plutôt que d'acheter une quantité trop importante d'ouvrages qu'il pourrait ne pas parvenir à écouler. Mohamed passe également commande en fonction de ce qu'il voit exposer sur les stands des kiosquiers et terrassiers installés à proximité. S'il constate que des titres sont fréquemment présents sur les étals, il ira s'approvisionner au marché de Derb Omar ou dans le quartier des Habous à Casablanca.

Les kiosquiers et les terrassiers, quant à eux, sont spécialisés dans la vente de journaux et de magazines. Les quelques livres présents sur leurs étals sont principalement en langue arabe, à caractère religieux ou universitaire. Mais on y trouve également des petits formats tels que les ouvrages de cuisine et ouvrages pratiques (santé, plantes médicinales, livres de savoir-vivre et d'actualité, etc.) étalés aux côtés des volumes du Coran, fréquemment vendus et achetés dans la rue. Ainsi, ni aux yeux des vendeurs ni aux yeux des fidèles, la dimension sacrée du livre saint n'est altérée par son contact direct avec les trottoirs.

De façon générale, le prix des ouvrages vendus par les kiosquiers et les terrassiers qui s'adressent, on l'a dit, principalement à un lectorat populaire, ne dépasse les 30 ou $40 \mathrm{dh}$, contrairement à ceux que commercialisent les libraires, et qui peuvent parfois excéder les $300 \mathrm{dh}$, en particulier les livres importés de France. C'est pourquoi il n'est pas rare que kiosquiers, terrassiers et bouquinistes photocopient des livres de France ou du Moyen-Orient et les relient à la façon de l'original, en conservant le format et la couverture.

La vente dans la rue ne requiert que des investissements réduits (peu ou pas de stocks). En outre, alors que les libraires sont séparés de la rue par une démarcation physique, les kiosquiers et les terrassiers ont établi des espaces ouverts par l'intermédiaire desquels les passants entrent en contact direct avec le livre, les journaux et les magazines pendant leurs déplacements.

Même si la frontière est parfois difficile à tracer, l'achat d'un ouvrage dans une librairie revêt une signification différente de celui d'un livre repéré à l'étalage d'un kiosque, sur le chemin du travail ou de la maison. Alors que le premier est souvent intentionnel de la part de lecteurs réguliers voire assidus, le second, propre aux lecteurs occasionnels, est généralement contingent ; ce qu'on appelle l'achat d'impulsion. L'emplacement et la morphologie des kiosques et des terrasses, comme le type de marchandises vendues expliquent cette différence : les journaux et les magazines attirent une nombreuse clientèle quotidienne qui s'intéresse également aux ouvrages, posés à plat sur le sol, dont le prix est abordable et la couverture attrayante. Ce constat a d'ailleurs incité les éditeurs à être particulièrement attentifs à l'apparence des livres : pour conquérir le marché populaire de la rue et voir leurs livres distribués par ses marchands, ils se doivent de travailler les couvertures de leurs collections.

\section{- Compétences et créativités professionnelles}

\section{La nécessité de la couleur}

Selon l'éditrice Leila Chaouni, à la tête de la maison Le Fennec, l'usage de la couleur pour les couvertures de ses publications est une nécessité non seulement pour attirer l'œil du client mais aussi pour faciliter le travail des marchands de rue à destination d'une clientèle dont une grande partie - en particulier les personnes âgées d'une quarantaine d'années et plus issues du milieu rural - est analphabète ou illettrée ${ }^{9}$ :

Au début, j'ai voulu faire dans la sobriété, au même titre que Gallimard, et n'utiliser que des couvertures blanches. Mais beaucoup m'avaient mise en garde : «À partir de quoi penses-tu que les kiosquiers et les terrassiers travaillent? Les couleurs sont leurs repères : passe-moi le [livre] vert! Mets-le [livre] rouge en évidence, il se vend bien! » Tu ne peux pas passer au blanc comme cela!

À quelques rares exceptions près, les éditeurs ont adopté la même charte graphique. Les illustrations sont très colorées et correspondent à des images abstraites : généralement des formes géométriques imbriquées les unes dans les autres. La neutralité de ces illustrations permet de les adapter à n'importe quel type de livres.

Les éditeurs peuvent ainsi réaliser des couvertures simples sans avoir à s'attarder sur la phase de conception qu'ils confient généralement à des maquettistes sous-traitants. Pour trouver leurs images, ils consultent de beaux livres d'art ou téléchargent des peintures sur internet qu'ils retravaillent à partir d'un logiciel d'infographie de retouche d'images. Les éditeurs les plus fortunés travaillent, quant à eux, avec des artistes à qui ils passent commande d' " images standard ", généralement peintes. D'autres encore veillent à ce que la couverture raconte en elle-même l'histoire du livre en utilisant des images figuratives. À leur façon, tous les éditeurs accordent une importance croissante à l'apparence de 


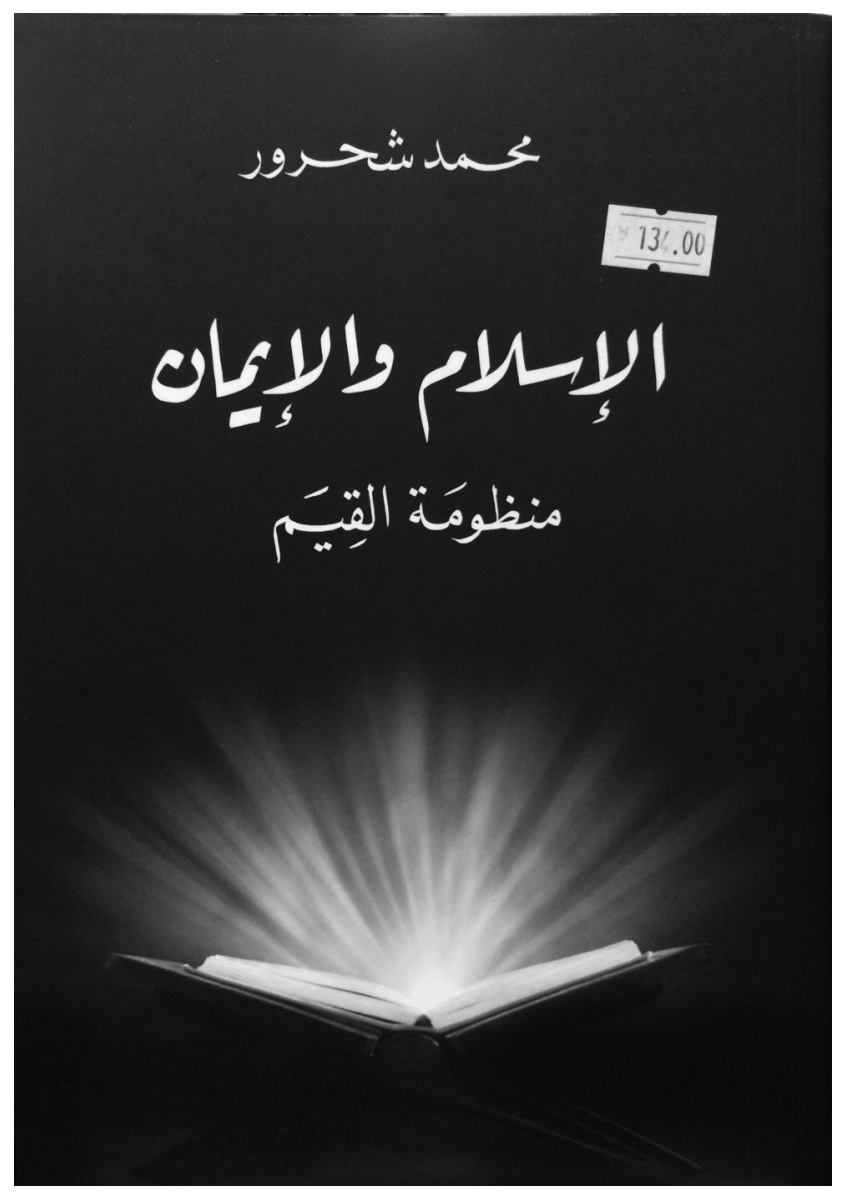

Photo 4 - Islam et foi, système des valeurs de Mohammed Shahrour.

leurs livres et ne se contentent plus de faire apparaitre le nom de l'auteur et le titre en calligraphie arabe sur un fond pâle (jaune, vert ou marron) comme c'était encore le cas au milieu des années 1990. Aujourd'hui, la grande majorité des couvertures de livres publiés au Maroc sont ornées de dessins colorés qui servent de référent aux marchands de livres, leur permettant soit d'établir un lien direct entre le titre et les illustrations, soit (quand ils ne savent pas lire) d'utiliser les couleurs comme repères.

À chaque commerçant sa technique : les marchands de rue ont recours à des moyens mnémotechniques divers pour exercer leur profession. Certains commerçants se réferent ainsi au format, aux reliures et autres types de matériaux pour présenter leur assortiment. D'autres se rapportent au nom de l'auteur, ou bien

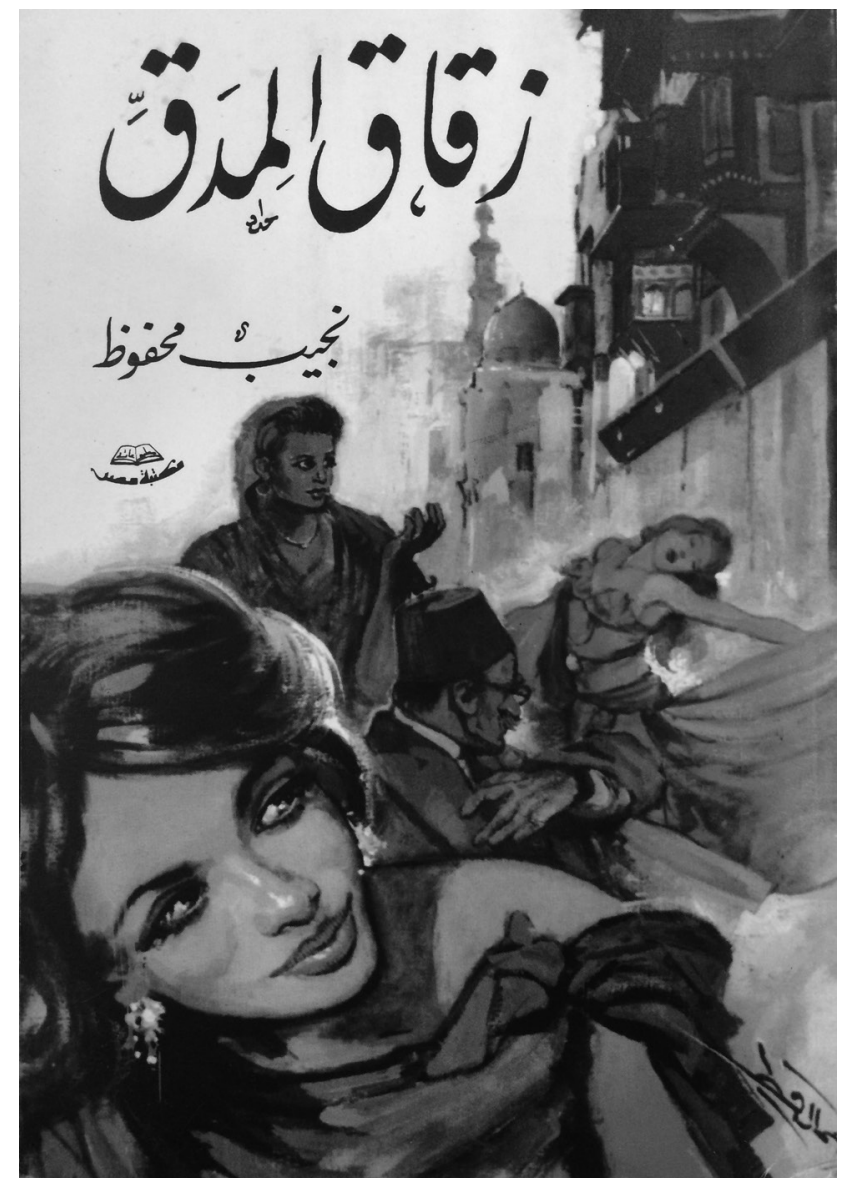

Photo 5 - Ruelles Midaq traduit en français en Passage des miracles de Naguib Mahfouz.

au titre et à son thème, associé tantôt à une couleur, tantôt à une autre. Par exemple, Mohamed, kiosquier, s'aide des reliures et des dorures pour différencier les livres islamiques contemporains des ouvrages religieux classiques, appelés mujallad (du mot jild : cuir, quoique ce matériau soit de plus en plus remplacé par le skai), exploitant un fonds éditorial clairement déterminé livres du patrimoine littéraire, linguistique et religieux arabes appelés les livres de turâth (turâth signifiant le legs culturel), volumes du Coran, commentaires et exégèses, poésie, littérature, etc.

\section{La matérialité du livre comme repère}

Ces ouvrages sont destinés à une diffusion de masse. Produits pour être lus par un large public, ils sont peu 
coûteux (entre 30 et $100 \mathrm{dh}$ ) tout en étant de bonne qualité. Il s'agit le plus souvent d'ouvrages d'une centaine de pages, de format moyen $(17 \times 24 \mathrm{~cm})$ et protégés par une couverture cartonnée, colorée et parfois pailletée. Les vendeurs différencient les livres islamiques contemporains à couverture cartonnée qu'ils appellent "kutub sulufan", des livres à couverture souple qu'ils nomment "kutub war'qa" (waraqa signifiant en arabe papier). De plusieurs types (romans, récits, essais, ouvrages religieux), les kutub war'qa ont une esthétique spécifique lorsqu'ils traitent de religion. À la différence des romans (riwâya), récits, et nouvelles (qissa) dont les premières de couverture représentent des dessins colorés, les kutub war'qa diel-dîn (relatifs à la religion) affichent une esthétique plus sobre : des écritures calligraphiques inscrites sur un fond homogène de couleur pastel (beige, vert, marron). Ainsi, les livres islamiques, de trois types (mujjalad, sulufan, war'qa) ont une dénomination liée à leur apparence utilisée par les marchands pour les distinguer entre eux et les tenir à l'écart d'autres types d'ouvrages.

Suivant les indications contenues sur la couverture (dessins, photos, peintures), l'écriture (calligraphiée ou non) et les matériaux, Mohamed regroupe les ouvrages par thèmes. Lorsque des photos de plats préparés - salades, couscous, tagines - décorent le livre, il ne doute pas qu'il s'agit d'un livre de cuisine.

Si le livre est protégé par une couverture souple et colorée, il sait qu'il s'agit d'un essai ou d'un ouvrage de littérature. En outre, le format, plus petit, et les couvertures fines signalent à Mohamed qu'il s'agit d'ouvrages égyptiens et libanais. Les livres marocains quant à eux présentent des spécificités liées aux méthodes d'impression locales : de format moyen $(17 \times 24 \mathrm{~cm}$ pour les essais, $14 \times 21$ pour les romans, les récits et les nouvelles), ils excèdent rarement les cent cinquante à deux cents pages et sont tous composés avec du papier de s80 grammes (exonéré d'impôt) et recouverts d'une couverture de s300 grammes, souple, plastifiée et mate. Une fois qu'il les a identifiés, Mohamed les regroupe par famille éditoriale plutôt que par thème, en s'aidant du logo de la maison d'édition. C'est ensuite aux clients d'identifier les sujets qui les intéressent dans la masse des ouvrages exposés devant eux, et aux éditeurs locaux de concevoir des logos reconnaissables.

Comme les kiosquiers et les terrassiers, les bouquinistes installés dans la médina ou dans les souks urbains ont également recours à la couleur et au format pour organiser leur stand. À Casablanca, on l'a dit, ils sont rassemblés dans un coin du souk de Derb Ghallef.
Comme d'autres marchés au Maroc, celui-ci est divisé en corps de métiers. À proximité des électriciens et des serruriers, la zone occupée par les bouquinistes comprend neuf boutiques de livres et de revues d'occasion ouvertes tous les jours de 9 heures à 18 h 30, sauf le vendredi, "jour de la prière » comme le soulignent les marchands. Chaque bouquiniste s'est spécialisé non seulement dans un domaine particulier (manuels scolaires, livres de littérature, revues, etc.) mais également dans une langue : certains proposent des livres d'occasion exclusivement en arabe, d'autres, plus nombreux, exclusivement en français. Ils ont une connaissance précise du fonds de leurs concurrents et n'hésitent pas à leur envoyer des clients.

Le stand d'Abderrahmane comme ceux de ses confrères est étroit et totalement exploité : tous les murs sont couverts d'étagères débordant d'ouvrages posés à l'horizontale ou à la verticale, de façon à utiliser l'espace au maximum. La prolifération d'ouvrages présentés en vrac transforme le livre en un objet de bazar. À la recherche d'un titre, j'essaie en vain de trouver une logique au classement. Quand je sollicite Abderrahmane, il répond aussitôt en pointant une zone de son stand: "Les livres Pocket, c'est là !». En fait, Abderrahmane a classé ses livres par collections en se fondant sur les formats et les couleurs: "Ici il y a les Pocket, là les J'ai Lu rouge, là les "grands romans" (déclare Abderrahmane en se référant à leur taille)... ", et sur les prix : "Les Harlequin coûtent $10 \mathrm{dh}$, les grands romans $25 \mathrm{dh}$, les Livres de poche, 20 dh et les Folio, 25 ou 30 dh ». Derrière l'impression d'extrême désorganisation, les ouvrages sont en fait rangés dans un ordre bien établi et structuré, surtout utile au bouquiniste.

Observer Abderrahmane renseigner les clients a été en effet l'occasion de constater qu'il connait parfaitement son fonds. Je lui demande pourquoi certains livres sont plus chers : "Parce qu'ils sont neufs !»-entendre " en bon état ». Sur la table la plus accessible de la boutique figurent les ouvrages d'auteurs célèbres comme Barbara Cartland, Agatha Christie, Driss Chraibi, Tahar Ben Jelloun et Naguib Mahfouz (traduit en français) qu'Abderrahmane cite aisément. Ainsi, il se réfere à trois critères principaux pour organiser sa boutique et hiérarchiser les prix de vente des ouvrages : la renommée de l'auteur; les caractéristiques de l'ouvrage (édition, collection, taille, couleur, couverture) et son état de conservation. Ces différents paramètres sont parfaitement maitrisés par Abderrahmane qui ne lit pas le français. À défaut de connaître le contenu des livres de son fonds, il a acquis au fil des années une parfaite 
maitrise de l'objet et de ses caractéristiques physiques. C'est grâce à ce savoir-faire qu'il parvient aujourd'hui à distinguer l'ensemble de ses ouvrages. Abderrahmane a par exemple constaté qu'un même auteur a souvent ses ouvrages publiés dans une même collection.

Comme Mohamed le kiosquier, il s'est davantage familiarisé avec le contenant des livres qu'avec leur contenu. La couleur et le format sont les critères principaux qui leur permettent à tous les deux de distinguer les livres, de les classer, de les ordonner, de leur attribuer un prix et donc d'exercer leur métier ${ }^{10}$.

Il est courant de voir les passants s'échanger des articles et en discuter face aux étalages de magazines et de livres déposés sur les trottoirs. La présence de l'imprimé dans l'espace public incite donc à la discussion autour de questions d'actualité, souvent politiques, morales et religieuses, animée ou non par les marchands. De cette manière, kiosquiers, terrassiers et bouquinistes sont des "personnages publics " au sens où Mitchell Duneier entend cette expression dans un ouvrage consacré aux vendeurs de livres et de magazines de rue d'un quartier new-yorkais à la fin des années 1990: comme des acteurs qui participent activement à la vie intellectuelle de leur ville et par là contribuent à y maintenir un certain ordre social [2000]. Le rôle crucial que les marchands de rue de Casablanca et de Rabat jouent dans la diffusion de l'écrit en en donnant l'accès à des personnes qui n'en sont pas familières ou en offrant la possibilité à des auteurs censurés de faire connaitre leur pensée corrobore ce constat. Par ailleurs, suivre les kiosquiers, terrassiers et bouquinistes dans leur travail a montré que le livre n'est pas seulement l'affaire de ceux qui lisent.
Des marchands analphabètes ou illettrés sont en charge de sa circulation dans la ville. Les éditeurs prennent en compte leurs pratiques de travail et adaptent la conception de leurs couvertures en conséquence. Plus précisément, ils sont attentifs aux manipulations singulières dont le livre fait l'objet de la part des terrassiers, kiosquiers et bouquinistes qui exploitent au maximum les caractéristiques physiques des livres pour structurer leur commerce. La matérialité de l'ouvrage occupe ainsi une fonction essentielle dans sa diffusion, de sorte qu'éditeurs, kiosquiers, terrassiers et bouquinistes participent ensemble à la fabrication de l'esthétique populaire du livre.

À partir des trottoirs où ils officient et des moyens dont ils disposent, ces marchands, qui entretiennent des liens étroits avec les points de vente de livres les plus formels, c'est-à-dire les librairies, ont su développer un rôle culturel fondamental dans la ville de Rabat et de Casablanca. Loin d'être dans une position de soumission à une autorité intellectuelle et institutionnelle, ils sont des «braconniers " [De Certeau, 1990] dans le sens où ils s'approprient et mettent à la disposition de tous des objets de connaissance de manière originale, y compris par le détournement et la ruse : «les savoirs n'existent pas sans les acteurs qui les pratiquent et ces pratiques, au-delà de leur finalité utilitaire immédiate, reflètent une subtile répartition des rôles, des compétences et de l'autorité dans une communauté donnée " [Jacob, 2011 : 20]. C'est cette répartition des rôles et des compétences dans la circulation du savoir à Rabat et à Casablanca que donne à voir l'observation de terrassiers, kiosquiers et bouquinistes officiant dans ces villes marocaines.

\section{Notes}

1. On utilise le même mot pour désigner le point de vente et le marchand.

2. Cette approche est au cœur de l'ouvrage à paraître en 2016: Anouk Cohen, Fabriquer le livre au Maroc, Paris, Karthala, "Terres et gens d'islam ».

3. À titre d'information, en 1998, Rabat et Casablanca comptent trois lecteurs arabophones pour un lecteur francophone [El Yazami, 1998]. Un ratio qui tend à évoluer en faveur de l'arabe en raison des politiques d'arabisation.

4. Pour une étude détaillée du monde du livre marocain - au sens où Howard Becker [1988] entend la notion de "Mondes de l'art": comme des réseaux d'acteurs qui coopèrent pour concourir à l'existence d'une œuvre -, se reporter à Cohen (2016, à paraître). Le monde du livre marocain a trois spécificités principales : il est jeune et en pleine transformation, urbain et dualiste. La constitution en cours d'un nouveau marché éditorial est d'abord liée aux récents progrès d'alphabétisation et de scolarisation dans le pays. Ces progrès, néanmoins, dissimulent de très importantes variations entre villes et campagnes ainsi qu'entre les capitales, fortement alphabétisées, et le reste du pays. Le monde du livre marocain se développe dans un cadre précis : le milieu urbain, en particulier l'axe Rabat-Casablanca. Ces deux villes situées au nord du pays, à une trentaine de kilomètres l'une de l'autre, abritent $90 \%$ des maisons d'édition ainsi que le réseau de librairies le mieux développé. À ce titre, elles ont constitué le lieu de mon analyse ethnographique. C'est également là que réside la plus grande part de la population alphabétisée qui lit et écrit dans deux langues principales : l'arabe et le français. En plus d'être jeune, en mutation et urbaine, l'édition locale est aussi dualiste, partagée entre les secteurs arabophone et francophone. Cette différenciation linguistique concerne l'ensemble de la chaîne du livre, des étapes de la fabrication à celles de la diffusion et de la réception. Rares en effet sont les éditeurs, les distributeurs (en gros et au détail) ou encore les auteurs et les lecteurs à travailler, voire à lire également dans les deux langues.

5. On nomme ainsi les musulmans ayant fait le pèlerinage à la Mecque.

6. Bien que l'amazigh soit historiquement la langue première du Maroc, il n'a longtemps pas eu de statut défini sinon qu'il fonctionne comme un langage véhiculaire au sein des communautés amazighophones rurales et vernaculaire 
parmi les communautés urbaines. Mais, depuis le discours royal d'Ajdir en 2001 et la création de l'Institut royal de la culture amazigh en 2008, l'amazigh bénéficie d'une reconnaissance formelle. Enfin, on l'a dit, à la suite des soulèvements populaires, Mohammed VI a annoncé, le 17 juin 2011, l'officialisation de l'amazigh comme deuxième langue du Maroc.

7. Le Sahara occidental est un territoire du nord-ouest de l'Afrique, bordé par le Maroc au nord, l'Algérie au nord-est, la Mauritanie à l'est et au sud, tandis que sa côte ouest donne sur l'Atlantique. Territoire non autonome selon l'ONU, cette ancienne colonie espagnole n'a toujours pas trouvé de statut définitif sur le plan juridique, plus de trente ans après le départ des Espagnols en 1976. Le territoire est revendiqué à la fois par le Maroc et par la République arabe sahraouie démocratique (RASD), fondée par le Front Polisario en 1976. Ce dernier mouvement, soutenu militairement, financièrement et diplomatiquement par l'Algérie, a pour objectif l'indépendance totale du Sahara occidental. Depuis le cessez-le-feu de 1991, le Maroc contrôle et administre environ $80 \%$ du territoire et maintient, derrière une longue ceinture de sécurité, le Front Polisario dans les $20 \%$ restant.

8. Cependant, l'écart entre ce qui doit être tenu secret et ce qui peut être révélé est devenu presqu'imperceptible. Du jour au lendemain, l'attitude des autorités oscille entre mansuétude et rigorisme. L'arbitraire domine. Selon un journaliste résidant à Casablanca : "Au moment de Hassan II, les lignes rouges étaient claires et infranchissables. Depuis le début du règne de Mohammed VI, le flou règne, on sait qu'elles existent mais on ne sait pas où elles se situent, on le comprend après coup, seulement une fois qu'on les a dépassées. " Pour plus d'informations à ce sujet, se reporter à Cohen [2011].

9. Selon le rapport officiel 50 ans de développement humain au Maroc (2006) l'analphabétisme touche près de $43 \%$ de la population âgée de dix ans et plus. Cependant, ce chiffre cache de profondes disparités. L'écart entre les villes et la campagne, par exemple, est de taille puisque l'analphabétisme touche $61 \%$ de la population rurale et $29 \%$ de la population urbaine. En outre, le taux d'analphabétisme est plus bas chez les jeunes et notamment les 10 à 15 ans : leur taux était de $36 \%$ en 1994, il est de $13 \%$ en 2007. Pour des statistiques détaillées, se reporter au rapport officiel 50 ans de développement humain au Maroc (2006).

10. Pour plus d'informations sur le rapport au livre et à l'écrit en milieu urbain marocain, se reporter à Cohen (2016, à paraitre).

\section{Références bibliographiques}

Becker Howard, 1988 [1982], Les Mondes de l'art, Paris, Flammarion.

Certeau Michel DE, 1990 [1980], L'Invention du quotidien, $t .1$ : Arts de faire, Paris, Gallimard, "Folio essais ".

Chartier Roger et Hans Jürgen Lusebrink, 1996, Colportage et Lecture populaire. Imprimés de large circulation en Europe. XVIXIX siècles, Paris, IMEC Editions.

Cohen Anouk, 2011, "La langue du silence dans le Maroc urbain contemporain", Revue d'Histoire des Religions, 3: 245-263.

Cohen Anouk, 2016, Fabriquer le livre au Maroc, Paris, Karthala, Coll. "Terres et gens d'islam », (à paraitre).

DuneIER Mitchell, 2000, Sidewalk, New-York, Farra, Straus and Giroux books.
El-Alaour Soraya, 2006, Les Réseaux du livre islamique. Parcours parisiens, Paris, CNRS éditions.

EL Yazami Abdelali, 1998, Enquête sur la lecture au Maroc, Rabat, Association marocaine des professionnels du livre.

Gonzalez-Quijano Yves, 2002 [1998], Les Gens du livre, Paris, CNRS éditions.

JACOB Christian, 2007, "Faire corps, faire lieu ", in Lieux de savoir. Espaces et communautés, t. 1, Jacob Christian (dir.), Paris, Albin Michel : 17-39.

JACOB Christian, 2011, "Introduction", Lieux de savoir. Les Mains de l'intellect, t. 2, Jacob Christian (dir.), Paris, Albin Michel.

Pouessel Stéphanie, 2008, "Écrire la langue berbère au Royaume de Mohammed VI : les enjeux politiques et identitaires du tifinagh au Maroc ", Revue des mondes musulmans et de la méditerranéee, 124 : 101-128.

50 ans de développement humain au Maroc et Perspectives 2025, 2006, Casablanca. 


\section{IBSTRACT}

Sidewalk Booksellers in Rabat and Casablanca (morocco). Distribution of Knowledge and Materiality of the Book

In Rabat and Casablanca, a large part of the editorial production, newspapers and magazines is directly distributed in the street, on sidewalks and in kiosks. The increased number of kiosquiers, terrassiers and second-hand book sellers (following the local terminology) indicated that their businesses are not marginal contrary to that their precarious appearance might suggest. The purpose of this article is to better understand the crucial role that these street traders, often illiterate or illiterate, play in the flow of writing in these Moroccan cities.

Keywords: Sidewalk booksellers. Materiality. Distribution of knowledge. Literacy. Morocco.

\section{ZUSAMMENFASSUNG}

Zeitschriften- und Buchhändler in Rabat und Casablanca. Wissensvermittlung und die Materialität von Büchern

In Rabat und Casablanca, jeweils Verwaltungs- und Wirtschaftshauptstadt Marokkos, wird ein großer Anteil der schriftlichen Produktion, Zeitungen und Zeitschriften direkt auf der Straße, auf Bürgersteigen und in Kiosken verkauft. Der hohe Anteil von Zeitschriften- und Buchhändlern auf der Straße zeigt auf, dass ihr Handel keine marginalisierte Verkaufsform ist, auch wenn die prekäre Lage dies vermuten ließe. Ziel dieses Artikels ist es, die zentrale Rolle dieser Straßenhändler, die häufig keine Schulbildung haben oder Analphabeten sind, für die Verbreitung von Schriften in den marokkanischen Städten aufzuzeigen.

Stichwörter: Buchhändler. Wissensvermittlung. Materialität. Analphabetismus. Marokko.

\section{RESUMEN}

Libreros de terrazas, de kioscos y libreros de viejo en Rabat y Casablanca. Circulación del saber y materialidad del libro

En Rabat y Casablanca, capitales administrativas y económicas de Marruecos, gran parte de la producción editorial, periódicos y revistas se despacha directamente en la calle, en las aceras y los kioscos. El número importante de libreros de terrazas, de kioscos y libreros de viejo (según la terminología empleada localmente) indica que sus negocios no constituyen un modo de difusión marginado, al contrario de lo que sugiere su aspecto precario. El objetivo de este artículo es definir mejor el papel crucial que estos vendedores de calle, muchas veces analfabetos o iletrados, cumplen en la circulación del escrito en estas ciudades marroquíes.

Palabras-clave: Vendedores de libros. Circulación del saber. Materialidad. Analfabetismo. Marruecos. 